\title{
Technical Note: Content Uniformity Measurement of an OTC Product Using a Dedicated Sample Preparation Station and a Fiber Optic UV Analysis System
}

Andrew Kielt*, Ishai Nir, Jeff Seely

e-mail: andrew.kielt@distekinc.com

Distek, Inc., North Brunswick, NJ.

\section{INTRODUCTION}

\section{(} ontent uniformity (CU) is performed using a wide range of techniques for sample preparation and analysis. Most are based on existing laboratory tools such as ultrasonic cleaners, orbital shakers, magnetic stirrers, or homogenizers not specifically optimized for CU sample preparation. Variation among different units in a lab, such as the power of different ultrasonic cleaners, the blade design of different homogenizers, or operator implementation of these can result in challenges in batch-to-batch reproducibility and OOS investigations. This problem is further exacerbated when a product is evaluated at multiple sites using different makes and models.

A solution that is specifically designed for CU sample preparation is needed. Such a CU sample prep station (PrepEngine, Distek, Inc., North Brunswick, NJ) is now available. It resolves most of the issues previously described with common sample preparation methods. It utilizes dedicated polypropylene sample processing vessels with a built-in, purpose-specific stirring blade, and operates at an adjustable speed ranging from 100 to 6000 RPM. The system also provides 10 individual stirring stations to match the USP Chapter <905> Uniformity of Dosage Units 10-unit assay requirement.

The CU sample prep station addresses the issue of reproducibility and length of time required for CU sample preparation. The reduced sample preparation time warrants equally rapid sample analyses. A fiber-optic UV spectrophotometer is ideal for this task. The fiber optic UV removes the need for dilution and filtration, both reducing total analysis time and avoiding the key sources of user errors. Fiber optic UV permits measurements directly in the sample prep container, with instant results.

\section{APPARATUS AND MATERIALS}

Content uniformity testing was conducted using the $\mathrm{CU}$ sample prep station and disposable $500-\mathrm{mL}$ sample prep vessels (PrepTubes, Distek, Inc). The sample solutions were prepared with deionized water and Tylenol regular strength (325 mg) tablets (Johnson \& Johnson). The samples were analyzed with a fiber optic UV (Opt-Diss 410, Distek, Inc.) equipped with $0.25-\mathrm{mm}$ pathlength probes (Arch Probe, Distek, Inc.). The analytical wavelength was $242 \mathrm{~nm}$, and the offset due to particles in the unfiltered samples was removed using an averaged baseline correction measured over the range of 325-350 nm. A reference standard for the fiber optic UV was prepared with acetaminophen reference standard material (Spectrum Chemical, New Brunswick, NJ).

\section{METHOD DEVELOPMENT}

The two parameters to be defined in establishing a CU sample preparation method are agitation speed and duration. To optimize these, $\mathrm{CU}$ testing was conducted using different speeds. The goal is to find the lowest agitation speed that will still yield guaranteed complete release in an acceptable test time. While the CU sample prep station is capable of operation at up to $6000 \mathrm{rpm}$, it is desirable to find a lower operating speed to maximize the number of samples that can be analyzed using each consumable sample prep vessel.

Tylenol regular strength tablets were added to 500$\mathrm{mL}$ sample prep vessels containing 500-mL deionized water before connecting to the $\mathrm{CU}$ sample prep station. The runs were stopped at different time points and the product absorbance inside the sample prep vessels was measured with the fiber optic UV to determine the percent extracted. Acetaminophen standard solution with a 0.651 $\mathrm{mg} / \mathrm{mL}$ concentration was prepared in deionized water to represent fully released product in $500 \mathrm{~mL}$. The UV spectrum of the acetaminophen standard was measured with the fiber optic UV. The percent extracted values were then calculated using the fiber optic UV software by comparing the sample absorbance to the standard absorbance. Measurement results are presented in the Table 1 and Figure 1. Based on these measurements, an agitation speed of 3000 RPM and a run time of $1.5 \mathrm{~min}$ were selected as the final method parameters. 
Table 1. Percent Extracted Results at Different Time Points and Speeds

\begin{tabular}{|c|c|c|c|c|}
\hline & \multicolumn{4}{|c|}{$\%$ Extracted } \\
\hline Total Time (s) & 500 RPM & 1000 RPM & 2500 RPM & 5000 RPM \\
\hline 0 & 0 & 0 & 0 & 0 \\
\hline 30 & 53.3 & 60.5 & 86.5 & 97.5 \\
\hline 60 & 80.2 & 90.8 & 101 & 101.4 \\
\hline 90 & 89.7 & 98.6 & 100.8 & 101.6 \\
\hline 120 & 95.3 & 100.4 & 101.1 & 101.4 \\
\hline 150 & 98.5 & & & \\
\hline 180 & 98.6 & & & \\
\hline 210 & 99.4 & & & \\
\hline 240 & 99.5 & & & \\
\hline 300 & 100.2 & & & \\
\hline
\end{tabular}

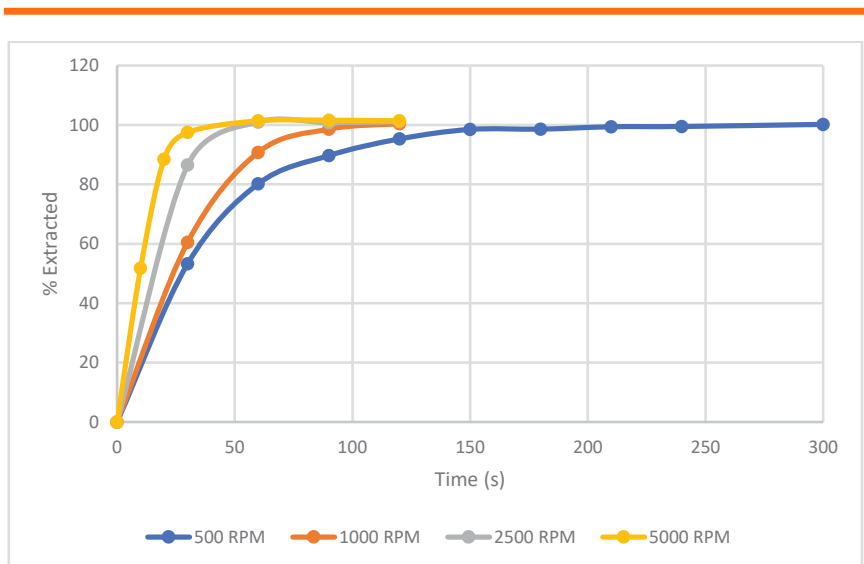

Figure 1: Percent extracted results as a fuction of agitation speed.

\section{RESULTS}

Ten 500-mL sample prep vessels were prepared by adding $500 \mathrm{~mL}$ deionized water and one Tylenol regular strength tablet before connecting to the $\mathrm{CU}$ sample prep station. At the end of the run, the fiber optic UV was again used to measure the final percent extracted in each sample prep vessel. Table 2 provides the measured percent extracted of the 10 tablets. The results shown were weight-corrected to eliminate the variation from tablet to tablet and highlight the measurement variability. The weight-corrected results show a mean $99.5 \%$ extracted and $\%$ RSD of $0.137 \%$. (For the uncorrected results, the mean is $99.5 \%$ and \%RSD is $0.66 \%$ ).
The limit of the acceptance value (AV) set in USP Chapter $<905>$ is less than $15.0 \%$ RSD for 10 units. For the data presented in Table 2, the corresponding AV value is 0.29 for weight-corrected values and 1.59 for uncorrected results, both well below the allowed value.

Table 2: Final Content Uniformity Results at 3000 RPM.

\begin{tabular}{|c|c|}
\hline Tablet & $\begin{array}{c}\text { Weight Corrected } \\
\text { (\% Extracted) }\end{array}$ \\
\hline 1 & 99.4 \\
\hline 2 & 99.4 \\
\hline 3 & 99.6 \\
\hline 4 & 99.5 \\
\hline 5 & 99.3 \\
\hline 6 & 99.6 \\
\hline 7 & 99.7 \\
\hline 8 & 99.4 \\
\hline 9 & 99.7 \\
\hline 10 & 99.5 \\
\hline
\end{tabular}

\section{CONCLUSION}

Dedicated CU sample preparation instrumentation provides a significant increase in efficiency and reliability. Paired with a fiber optic UV, the combination creates a fully robust measurement process spanning sample preparation to analysis, with the important additional benefit of significantly shorter total test times and instant results. Some products tested using this solution outside of this technical note, such as oxycodone with "crush-proof" coatings, were able to be measured in $10 \%$ of the previous time. The simplicity of method development, consistency of measurement, and time savings were demonstrated in actual measurements using a representative OTC product. 